\title{
A RANGE-ONLY MULTIPLE TARGET PARTICLE FILTER TRACKER
}

\author{
Volkan Cevher, Rajbabu Velmurugan, and James H. McClellan \\ Georgia Institute of Technology \\ Atlanta, GA 30332-0250
}

\begin{abstract}
We propose a particle filter tracker to track multiple maneuvering targets using a batch of range measurements. The state update is formulated through a locally linear motion model and the observability of the state vector is proved using geometrical arguments. The data likelihood treats the range observations as an image using template models derived from the state update equation, and incorporates the possibility of missing data as well as spurious range observations. The particle filter handles multiple targets, using a partitioned statevector approach. The filter proposal function uses a Gaussian approximation of the full-posterior to cope with target maneuvers for improved efficiency. By treating the range measurements as images and using smoothness constraints, the particle filter is able to avoid the data association problems. Computer simulations demonstrate the performance of the tracking algorithm.
\end{abstract}

\section{INTRODUCTION}

Radar range tracking problem is a challenging signal processing problem that has attracted very little interest in the literature [1-3]. This tracking problem is usually formulated using state-space models, where the target's motion is approximated as local linear (e.g., constant velocity) and the observations are temporal snapshots of radar range and range-rate estimates. Then, to estimate the state vector consisting of the target's position and velocity using range-only measurements, a mobile platform must be used that executes known maneuvers for system observability [2]. Otherwise, multiple beacons must be used to track the state vector by virtue of triangulation [3].

In this paper, we present a particle filter algorithm to track a state vector that consists of the target direction-of-arrival (DOA) $\theta(t)$, the logarithm of the target range $R(t)$, the target speed $v(t)$, and the target heading $\phi(t)$, using a batch of range-only measurements, obtained at a stationary sensor. The angles are measured counterclockwise with respect to the $x$-axis. We prove that the particle filter state vector is observable given at least three range measurements under rotational and planar-symmetrical ambiguities. Our proof makes use of the Stewart's triangle theorem in geometry.

The motivation for the state vector of the particle filter is the low power RF sensor, implemented at the University of Florida that transmits a microwave signal to determine the range, the velocity, and the size of the detected targets [4]. The sensor is capable of providing range estimates at $32 \mathrm{~ms}$ intervals with a range resolution of approximately $2 \mathrm{~m}$ on a range-Doppler map. Up to $100 \mathrm{~m}$, the current system is capable of producing range estimates for multiple ground vehicles as well as human targets. The radar hardware is envisioned

Prepared through collaborative participation in the Advanced Sensors Consortium sponsored by the U. S. Army Research Laboratory under the Collaborative Technology Alliance Program, Cooperative Agreement DAAD1901-02-0008 to have a larger detection range with hemispherical coverage in the future. Note that the filter equations in this paper are developed using range-only measurements so that it is also applicable to amplitude tracking problems. Additional velocity measurements or range-rate measurements can be easily incorporated through the data likelihood equation via independence assumptions.

The particle filter uses a batch of range measurements to determine the state vector, based on an image template matching idea. The template matching idea is very effective when accurate models are available [5]. In our problem, a temporal range image is first formed, when a batch of range measurements are received. Then, candidate image templates are formed by using the state update function and the target state vectors. By determining the best matching image template, the target state vectors are determined. It is assumed that the range measurements are normally distributed around the true range measurements, with constant data miss-probability and clutter density.

The presence of multiple targets increases the tracking complexity, because the received data must be sorted for each target. Since the particle filter treats its range-only measurements as an image, the data association and ordering problems are naturally alleviated. To handle multiple targets, the particle filter uses a partitioning approach, where a particle consists of the concatenation of multiple target state vectors. We use the probabilistic data association methods to estimate the states by summing over all the association hypothesis weighted by the likelihood probabilities [6]. The particle filter proposal function independently proposes particles for its partitions by using a Gaussian approximation of the full posterior density for efficiency. Hence, the presented particle filter is robust against the curse of dimensionality problem [7], when the number of targets increase.

To derive the proposal function, the multi target posterior density is approximately factorized for each target. Then the Laplace's method is used to approximate each partition posterior by a Gaussian around its mode [8]. We calculate the partition modes using a robust Newton-Raphson recursion with a backtracking step size selection that imposes smoothness constraints on the target motion [9]. This approach is similar to the one for an angle-only tracking filter [10].

\section{DATA MODELS}

\subsection{State Update Model}

The filter state vector $\mathbf{x}_{t}=\left[x_{1}^{T}(t), x_{2}^{T}(t), \cdots, x_{K}^{T}(t)\right]^{T}$ consists of the concatenation of the partition vectors $x_{k}(t)$ for each target, indexed by $k, k=1, \ldots, K$. Each partition has the corresponding target motion parameters $x_{k}(t) \triangleq\left[\theta_{k}(t), R_{k}(t), v_{k}(t), \phi_{k}(t)\right]^{T}$, where $\theta_{k}(t)$ is the DOA, $R_{k}(t)$ is the logarithm of the range, $v_{k}(t)$ is the speed, and $\phi_{k}(t)$ is the heading direction. For notational convenience, the logarithm of the range is used in the state vector because the range errors are modeled multiplicative. 


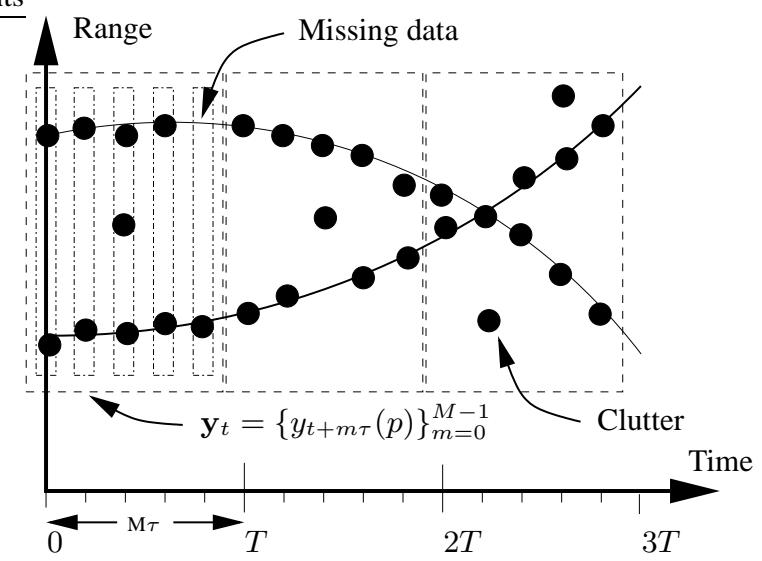

Fig. 1. Observation model using batch of radar range measurements. Note that the range measurements are not necessarily ordered. However, the image based observation approach provides a natural ordering, when targets are being tracked by the particle filter.

The state-update equation can be derived from the geometry imposed by a motion model on the state vector. In this paper, we model the target motion with a locally constant velocity model. The resulting state-update equation is nonlinear:

$$
x_{k}(t+T)=h_{T}\left(x_{k}(t)\right)+u_{k}(t),
$$

where $u_{k}(t) \sim \mathcal{N}\left(0, \Sigma_{u}\right)$ with $\Sigma_{u}=\operatorname{diag}\left\{\sigma_{\theta, k}^{2}, \sigma_{r, k}^{2}, \sigma_{v, k}^{2}, \sigma_{\phi, k}^{2}\right\}$ and $h_{T}\left(x_{k}\right)=$

$$
\left[\begin{array}{c}
\tan ^{-1}\left\{\frac{e^{R_{k}} \sin \theta_{k}+T v_{k} \sin \phi_{k}}{e^{R_{k} \cos \theta_{k}+T v_{k} \cos \phi_{k}}}\right\} \\
\frac{1}{2} \log \left\{e^{2 R_{k}}+T^{2} v_{k}^{2}+2 T e^{R_{k}} v_{k} \cos \left(\theta_{k}-\phi_{k}\right)\right\} \\
v_{k} \\
\phi_{k}
\end{array}\right]
$$

\subsection{Observation Model}

The observations $\mathbf{y}_{t}=\left\{y_{t+m \tau}(p)\right\}_{m=0}^{M-1}$ consists of range estimates from a radar sensor at each batch index $m$. This observation model can be visualized as shown in Fig. 1. The radar returns over the time-interval $\tau$ are used to estimate, possibly multiple, target ranges. A batch of $M$ such range estimates are used by the particle filter to estimate target state every $T=M \tau$ seconds. It is assumed that the batch of measurements are normally distributed around the true target ranges with variance $\sigma_{r}^{2}$ and a constant data miss probability matrix $\kappa$. This batch may include spurious peaks due to clutter that are Poisson distributed with rate $\lambda$.

We derive the data-likelihood function using the joint probabilistic data association arguments found in [6]. Consider the output of one batch period $\mathbf{y}_{m}=y_{t+m \tau}(p)$, where $p=0,1, \ldots, P_{m}$ for each $m$. The range measurements $\mathbf{y}_{m}$ may belong to none, or some combination, or all of the targets in the particle filter partitions. Hence, define a set $\mathcal{I}_{n}$ that consists of $n$-unordered combination of all $K$ partitions of the particle filter state vector: $\mathcal{I}_{n} \in\left\{{ }_{K} \mathcal{C}_{n}\right\}$, where ${ }_{K} \mathcal{C}_{n}$ is number of ways of picking $n$-unordered outcomes from $K$ possibilities.

Each element of $\mathcal{I}_{n}$ has $n$ numbers, and there are a total of ${ }_{K} \mathcal{C}_{n}$ elements. For example, when $K=3$ and $n=2$, then $\mathcal{I}_{2}=$ $\{\{1,2\},\{1,3\},\{2,3\}\}$, each element referring to subset of the individual partitions of the particle state vector. We refer to the in- dividual elements of this set using the notation $\mathcal{I}_{n}(j)$, where $j=$ $1, \ldots,{ }_{K} \mathcal{C}_{n}$. Hence, $\mathcal{I}_{2}(2)=\{1,3\}$. Then, denote ${ }_{n} \mathbf{x}_{t}(j) \in$ $\left\{x_{i}(t) \mid i \in \mathcal{I}_{n}(j), x_{i}(t) \in \mathbf{x}_{t}\right\}$ as a single realization from $\mathcal{I}_{n}$. Using the same example above, we have ${ }_{2} \mathbf{x}_{t}(3)=\left[x_{2}^{T}(t), x_{3}^{T}(t)\right]^{T}$ $=\left[\hat{x}_{1}^{T}(t), \hat{x}_{2}^{T}(t)\right]^{T}$. The elements of the vector ${ }_{n} \mathbf{x}_{t}(j)$ are shown, in order, by $\hat{x}_{i}(t)(i=1, \ldots, n)$, given the parameters $n$ and $j$.

We denote $\pi_{n, j}\left(\mathbf{y}_{m}\right)=p\left(\left.\mathbf{y}_{m}\right|_{n} \mathbf{x}_{t}(j)\right)$ as the probability density function of the data, where only $n$ range measurements belong to the targets defined by the partitions of ${ }_{n} \mathbf{x}_{t}(j)$. Hence, when $n=0$, all data is due to clutter:

$$
\pi_{0,1}\left(\mathbf{y}_{m}\right)=\lambda^{P_{m}}
$$

The probability density $\pi_{n, j}\left(\mathbf{y}_{m}\right)$ can be calculated by noting that (i) there are $P_{m} ! /\left(P_{m}-n\right)$ ! ordered ways of choosing range measurements to associate with the $n$-subset partitions, and (ii) the remaining $\left(P_{m}-n\right)$ range measurements are explained by the clutter:

$$
\pi_{n, j}\left(\mathbf{y}_{m}\right)=\frac{\left(P_{m}-n\right) ! \lambda^{P_{m}-n}}{P_{m} !} \sum_{p_{1} \neq p_{2} \neq \ldots \neq p_{n}}^{P_{m}} \prod_{i=1}^{n} \psi_{t, m}\left(p_{i} \mid \hat{x}_{i}\right),
$$

where the function $\psi$ is the following Gaussian distribution:

$\psi_{t, m}\left(p_{i} \mid x_{i}\right)=\frac{1}{\sqrt{2 \pi} \sigma_{r}} \exp \left\{-\frac{\left(h_{m \tau}^{R}\left(x_{i}(t)\right)-y_{t+m \tau}\left(p_{i}\right)\right)^{2}}{2 \sigma_{r}^{2}}\right\}$,

where the superscript $R$ on the state update function $h$ refers only to the log-range component of the state update and $\sigma_{r}^{2}$ is determined by the radar hardware capabilities.

Given the densities $\pi_{n, j}$, the observation density function can be constructed as a combination of all the target association hypotheses. Hence, by adding mixtures that consist of the data permutations and the partition combinations, we derive the observation density:

$$
p\left(\mathbf{y}_{t} \mid \mathbf{x}_{t}\right)=\prod_{m=0}^{M-1} \sum_{n=0}^{K} \frac{\kappa_{n, K}}{{ }_{K} \mathcal{C}_{n}} \sum_{j=1}^{K \mathcal{C}_{n}} \pi_{n, j}\left(\mathbf{y}_{m}\right)
$$

In (6), the parameters $\kappa_{n, K}, \sum_{n} \kappa_{n, K}=1$, are the elements of a detection (or confusion) matrix. For example, when $K=2, \kappa_{0,2}$ is the probability that no target range estimate is in the radar output, whereas $\kappa_{1,2}\left(\kappa_{2,2}\right)$ implies that 1 (2) target range(s) are present in the radar output. These fixed values have to be provided by the user. However, they should be changed adaptively to improve robustness of the particle filter output. For example, when two partitions $k_{1}$ and $k_{2}$ have close range tracks and are about to cross, we change the confusion matrix to indicate the possibility that one of the targets will likely be missed.

\subsection{Approximate Partition Posterior Functions}

In our problem of range-only multiple target tracking, the proposal function poses difficult challenges because (i) the state vector dimension is proportional to the number of targets $K$, hence the number of particles to represent posterior can increase significantly as $K$ increases (the curse of dimensionality), (ii) in many cases, the targets maneuver, hence full posterior approximations are required for robust tracking, and (iii) for full posterior approximations, robustly determining the range-only data-likelihood is rather hard.

In our problem, we can approximately factor out the tracking posterior to exploit the computational advantage of the partitioned sampling. Note that in our case, the target dynamics can already 
be factored out because we assume the targets are moving independently. Unfortunately, the observation density does not factor out, because the observed radar range data cannot be immediately associated with any of the partitions. However, for a given partition, if we assume that the data is only due to that partition and clutter (hence, the range measurements corresponding to other partitions are treated as clutter), we can do the following approximate factorization on the observation likelihood (6): $p\left(\mathbf{y}_{t} \mid \mathbf{x}_{t}\right) \approx \prod_{k=1}^{K} p\left(\mathbf{y}_{t} \mid x_{k}(t)\right)=$

$$
\prod_{k=1}^{K} \prod_{m=0}^{M-1}\left\{\kappa_{0,1} \lambda^{P_{m}}+\kappa_{1,1} \lambda^{P_{m}-1} \sum_{p=1}^{P_{m}} \frac{\psi_{t, m}\left(p \mid x_{k}\right)}{P_{m}}\right\} .
$$

Hence, for our problem, each approximate partition posterior is given by

$$
q_{k}\left(x_{k}(t) \mid \mathbf{y}_{t}, x_{k}(t-T)\right) \propto p\left(\mathbf{y}_{t} \mid x_{k}(t)\right) p\left(x_{k}(t) \mid x_{k}(t-T)\right),
$$

where $p\left(\mathbf{y}_{t} \mid x_{k}(t)\right)$ is given in (7). Note that (7) is not used as the data-likelihood of the particle filter. The above approximate factorization of the data-likelihood is to make use of the partitioned sampling strategy to propose particles. To calculate the particle filter weights, the full posterior uses the observation density (6).

\subsection{Particle Filter Proposal Function}

To capture target maneuvers effectively, we use the current observed data to propose the filter's particle support. The filter uses Laplace's method to approximate $p\left(\mathbf{y}_{t} \mid x_{k}(t)\right)$ in (8) and thereby derives the partition proposal functions of the particle filter, where $x_{k}(t) \sim$ $g_{k}\left(x_{k}(t) \mid \mathbf{y}_{t}, x_{k}(t-T)\right)$. Laplace's method is an analytical approximation of probability density functions based on a Gaussian approximation of the density around its mode, where the inverse Hessian of the logarithm of the density is used as a covariance approximation [11]. It can provide adequate approximations to posteriors that are as accurate and sometimes more accurate than the approximations based on third-order expansions of the density functions [8].

Laplace's approximation requires the calculation of the data statistics. The Laplacian approximation is described in [10] and is implemented with the Newton-Raphson recursion with backtracking step size selection for computational efficiency. The final expression for the partition proposal functions is given by

$$
g_{k}\left(x_{k}(t) \mid \mathbf{y}_{t}, x_{k}(t-T)\right) \sim \mathcal{N}\left(\mu_{g}(k), \Sigma_{g}(k)\right),
$$

where the Gaussian density parameters are

$$
\begin{aligned}
& \Sigma_{g}(k)=\left(\Sigma_{y}^{-1}(k)+\Sigma_{u}^{-1}\right)^{-1}, \\
& \mu_{g}(k)=\Sigma_{g}(k)\left(\Sigma_{y}^{-1}(k) x_{k, \text { mode }}+\Sigma_{u}^{-1} h_{T}\left(x_{k}(t-T)\right)\right) .
\end{aligned}
$$

The vector $x_{k \text {,mode }}$ is the mode of $p\left(\mathbf{y}_{t} \mid x_{k}(t)\right)$, and $\Sigma_{y}^{-1}(k)$ is the Hessian of $p\left(\mathbf{y}_{t} \mid x_{k}(t)\right)$ at $x_{k, \text { mode }}$, calculated under the motion smoothness constraints, as described in [10].

\subsection{Observability of the State Vector}

Figure 2 illustrates that it is possible to determine $v$ and $\theta-\phi$ given three range measurements. This follows from the Stewart's theorem that can be proved by using the law of cosines on the triangles $\triangle O A B$ and $\triangle O A C$. Note that $\theta$ and $\phi$ can not be determined uniquely given only the range measurements: the trajectories defined by $A B C, A_{1} B_{1} C_{1}$, and $A_{2} B_{2} C_{2}$ can result in the same range measurements $\left\{r_{1}, r_{2}, r_{3}\right\}$.

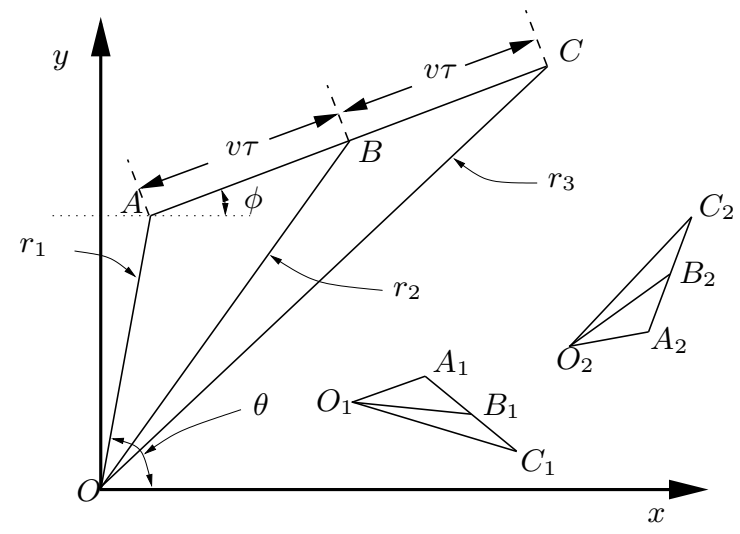

Fig. 2. The speed can be calculated as $v=\frac{1}{\tau} \sqrt{\frac{1}{2}\left(r_{1}^{2}+r_{3}^{2}\right)-r_{2}^{2}}$. Triangles $\triangle O_{1} A_{1} C_{1}$ and $\triangle O_{2} A_{2} C_{2}$ are scaled versions of $\triangle O A C$ that demonstrate the rotational and planar symmetric ambiguities, respectively. They are scaled down due to lack of space.

Table 1. Simulation Parameters

\begin{tabular}{|c|c|c|c|c|c|c|c|}
\hline \hline$N$ & $T$ & $M$ & $\sigma_{r}$ & $\sigma_{u, \theta}$ & $\sigma_{u, r}$ & $\sigma_{u, v}$ & $\sigma_{u, \phi}$ \\
\hline \hline 200 & 1 & 10 & 1 & $1^{\circ}$ & 0.1 & 0.1 & $10^{\circ}$ \\
\hline \hline
\end{tabular}

\section{SIMULATIONS}

This section presents simulation results to demonstrate the performance of the range-only multiple target tracker. Both single and multiple target tracking scenarios are considered. The synthetic range measurement data is obtained using the constant velocity motion of the targets. The data is perturbed using a zero mean Gaussian noise with variance $\sigma_{r}^{2}$. The simulation parameters that generated Figs. 3 and 4 are shown in Table 1.

Figure 3 shows the tracking results obtained for a single target tracking scenario. The target moves with a constant speed of $14 \mathrm{~m} / \mathrm{s}$. The radar range measurements with clutter are input to the filter unordered. The tracker is initialized with $N$ particles generated by adding noise (Table 1 ) to the true target state at $t=0$ s. The tracker can associate the range measurements related to the target motion and can provide accurate temporal estimates of the single target state. The filter heading estimates lags the true target heading due to our assumption of the constant velocity motion. Also, the tracking results for two targets are shown in Fig. 4. The targets move with a constant speed of $14 \mathrm{~m} / \mathrm{s}$ and $14.5 \mathrm{~m} / \mathrm{s}$. The particle filter was able to resolve the data association issues at times $t=3 \mathrm{~s}$ and $t=30 \mathrm{~s}$. The motion estimates are unique due to correct initialization.

The estimates obtained using range-only measurements can be further improved by using additional measurements. For example, define $\alpha_{k}=v \cos \left(\theta_{k}-\phi_{k}\right)$ as the range-rate. Then, we can treat the additional range rate measurements as an independent observation and calculate $p\left(\boldsymbol{\alpha} \mid \mathbf{x}_{t}\right), \boldsymbol{\alpha}=\left[\alpha_{1}, \ldots, \alpha_{K}\right]^{T}$, by using the same joint density association approach as (6). The range-rate measurements are then incorporated at the weighting stage of the particle filter algorithm:

$$
w_{t}^{*(i)}=w_{t-T}^{(i)} \frac{p\left(\mathbf{y}_{t} \mid \mathbf{x}_{t}^{(i)}\right) p\left(\mathbf{x}_{t}^{(i)} \mid \mathbf{x}_{t-T}^{(i)}\right)}{\prod_{k} g_{k}\left(x_{k}^{(i)}(t) \mid \mathbf{y}_{t}, x_{k}^{(i)}(t-T)\right)} p\left(\boldsymbol{\alpha} \mid \mathbf{x}_{t}^{(i)}\right),
$$



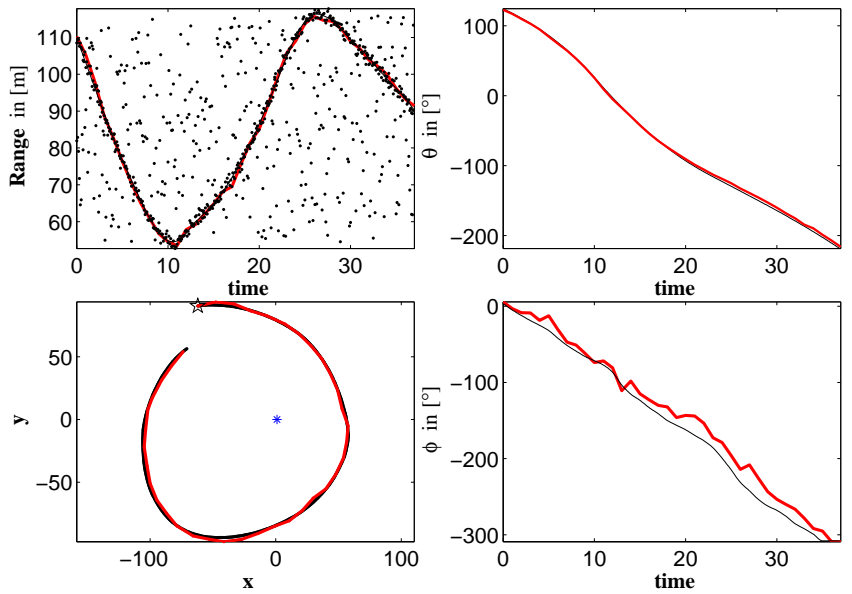

Fig. 3. Single target tracking example. Black dots in the top left panel are range measurements. The pentagram and the star indicate the target starting position and the radar position, respectively.

where $w^{*}$ is the unnormalized weight. The range-rate measurements further improve the tracking estimates as shown in Fig. 5, where a higher observation variance $\sigma_{r}^{2}=4$ is used with $\sigma_{\alpha}^{2}=1$.

\section{CONCLUSIONS}

In this paper, we present a range-only multiple target particle filter tracker based on a batch measurement model. The radar range measurement batches are treated as an image to naturally handle the data association and ordering issues. The presence of multiple targets is handled using a partition approach. The observation likelihoods are calculated jointly and are assigned by using the templates created by the state vectors and the state update equation. The current form of the filter can also be used for amplitude tracking problems.

\section{REFERENCES}

[1] Branko Ristic, Sanjeev Arulampalam, and James McCarthy, "Target motion analysis using range-only measurements: algorithms, performance, and application to ISAR data," Elsevier Signal Processing, vol. 82, pp. 273-296, 2002.

[2] T. L. Song, "Observability of target tracking with range-only measurements," IEEE Journal of Oceanic Engineering, vol. 24, pp. 383-387, July 1999.

[3] R. Doraiswami, "A novel Kalman filter-based navigation using beacons," IEEE Transactions on Aerospace and Electronic Systems, vol. 32, pp. 830-840, April 1996.

[4] R. T. Damarla, V. Hao, C. Reiff, and J. Kurtz, "Fusion of acoustic and radar data for tracking vehicles," in Military Sensing Symposium, Laurel, MD, 22-25 August 2005.

[5] M. Isard and A. Blake, Active Contours, Springer, 2000.

[6] Y. Bar-Shalom and T. Fortmann, Tracking and Data Association, Academic-Press, 1988.

[7] D. Crisan and A. Doucet, "A survey of convergence results on particle filtering methods for practitioners," IEEE Trans. on Signal Processing, vol. 50, no. 3, pp. 736-746, March 2002.
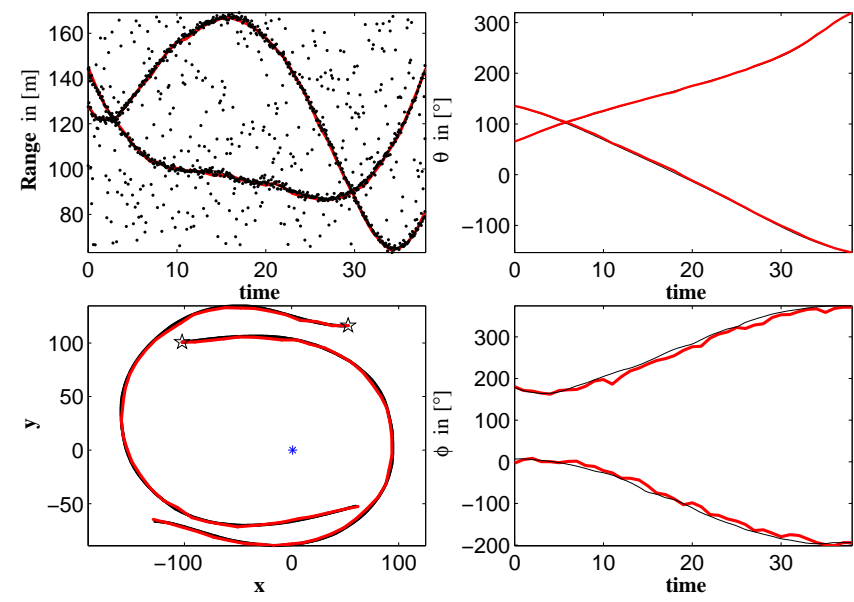

Fig. 4. Multiple target tracking. The heading estimates have more oscillations than the DOA estimates, because they are more sensitive to the noise in the range. The filter does a good job in associating the data with the targets because of the joint density approach.
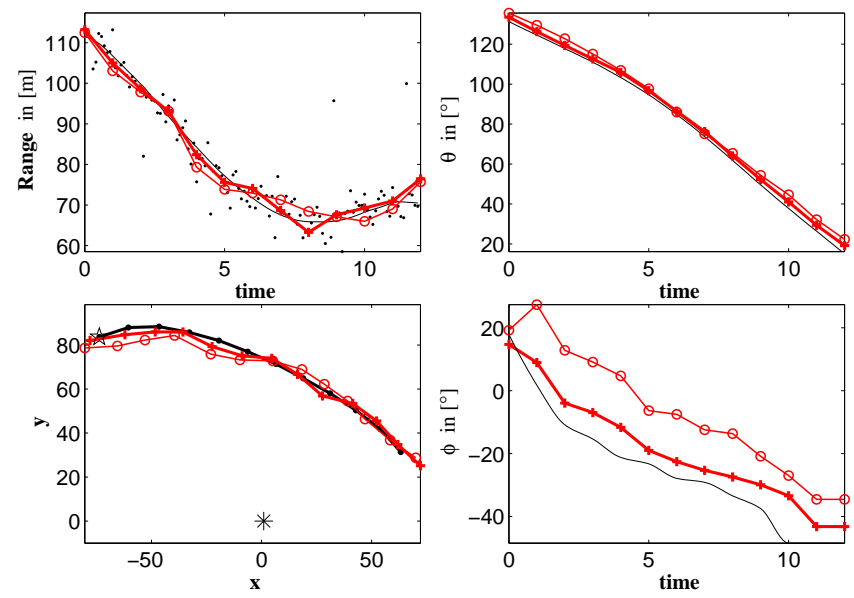

Fig. 5. Additional range-rate measurements can be incorporated at the weighting stage of the filter, without changing the filter. Heading estimates with range-rate measurements, marked with + , are closer to the true values than without the range-rate measurements, marked with $\circ$.

[8] L. Tierney and J. B. Kadane, "Accurate approximations for posterior moments and marginal densities," Journal of the American Statistical Association, , no. 81, pp. 82-86, 1986.

[9] J.E.Dennis Jr. and R.B. Schnabel, Numerical Methods for Unconstrained Optimization and Nonlinear Equations, Society for Industrial and Applied Mathematics, Philadelphia, PA, 1996.

[10] V. Cevher and J. H. McClellan, "An acoustic multiple target tracker," in IEEE SSP 2005, Bordeaux, FR, 17-20 July 2005.

[11] A. Gelman, J. B. Carlin, H. S. Stern, and D. B. Rubin, Bayesian Data Analysis, Chapman Hall/CRC, 2004. 